\title{
Clinical significance of serum anti-p53 antibody expression following curative surgery for colorectal cancer
}

\author{
KENSUKE KUMAMOTO ${ }^{1,2}$, HIDEYUKI ISHIDA ${ }^{1}$, KOKI KUWABARA ${ }^{1}$, KUNIHIKO AMANO ${ }^{1}$, \\ NORIYASU CHIKA ${ }^{1}$, NORIMICHI OKADA ${ }^{1}$, TOMONORI OHSAWA ${ }^{1}$, \\ YOUICHI KUMAGAI $^{1}$ and KEIICHIRO ISHIBASHI ${ }^{1}$ \\ ${ }^{1}$ Department of Digestive Tract and General Surgery, Saitama Medical Center, Saitama Medical \\ University, Kawagoe, Saitama 350-8550; ${ }^{2}$ Department of Coloproctology, Aizu Medical Center, \\ Fukushima Medical University, Aizuwakamatsu, Fukushima 969-3492, Japan
}

Received April 3, 2017; Accepted August 3, 2017

DOI: $10.3892 / \operatorname{mco} .2017 .1368$

\begin{abstract}
The aim of the present study was to investigate the usefulness of serum anti-p53 antibody (Ap53Ab) measurement for the diagnosis of colorectal cancer (CRC), and the clinical significance of the association between Ap53Ab expression and survival rate. Ap53Ab, carcinoembryonic antigen (CEA) and carbohydrate antigen (CA)19-9 were measured by ELISA in $674 \mathrm{CRC}$ patients and 115 healthy volunteers (control group). The half-life time of Ap53Ab and CEA was calculated. The association between positive Ap53Ab expression and clinicopathological characteristics, including survival rate, was analyzed. Of the 674 CRC patients, 195 (28.9\%) were positive for Ap53Ab expression, while the positive rates of CEA and CA19-9 level were 39.9 and $16.9 \%$, respectively. Positivity for Ap53Ab alone was observed in 94 patients (13.9\%), whereas the positivity rate of any markers examined was $58.7 \%$. The mean half-life of Ap53Ab and CEA was 30.7 and 11.3 days, respectively. Positive expression of Ap53Ab was significantly associated with the depth of tumor invasion $(\mathrm{P}<0.001)$, lymph node metastasis $(\mathrm{P}=0.024)$, stage $(\mathrm{P}<0.001)$ and $\mathrm{CEA}$ level $(\mathrm{P}=0.005)$. No significant correlation between Ap53Ab expression and poor survival rate was observed. The positive rate of Ap53Ab was higher compared with that of CEA and CA19-9 in early-stage CRC. The combination of these markers improved the diagnostic yield of CRC up to $\sim 60 \%$. Furthermore, Ap53Ab expression was associated with lymph node metastasis, but not with shorter survival. These results indicated that the measurement of Ap53Ab may contribute to increased rate of detection
\end{abstract}

Correspondence to: Dr Kensuke Kumamoto, Department of Coloproctology, Aizu Medical Center, Fukushima Medical University, 21-2 Maeda, Tanisawa, Kawahigashi, Aizuwakamatsu, Fukushima 969-3492, Japan

E-mail:kumamotk@fmu.ac.jp

Key words: anti-p53 antibody, carbohydrate antigen 19-9, carcinoembryonic antigen, colorectal cancer, tumor marker of CRC, particularly in patients with early-stage disease, in clinical practice.

\section{Introduction}

Colorectal cancer (CRC) is the most common cancer and the second leading cause of cancer-related mortality in Japan. Tumor markers, including carcinoembryonic antigen (CEA) and carbohydrate antigen (CA)19-9, have been used for the screening of CRC in clinical practice. These markers are useful for monitoring cancer recurrence and the efficacy of chemotherapy in advanced CRC patients with positive expression of CEA or CA19-9. However, the sensitivity of these markers is very low in early-stage CRC. Therefore, the development of novel molecular markers is required, using blood testing as a non-invasive method. The serum anti-p53 antibody (Ap53Ab) has been applied as a novel tumor marker for the detection of several cancers, including esophageal cancer $(1,2)$, breast cancer $(1,3)$ and CRC $(1,4,5)$, from 2007 onwards in Japan. Mutations of the TP53 gene, which were detected in half of CRC cases (6), were strongly associated with carcinogenesis. The accumulation of mutated-TP53 protein induces the synthesis of Ap53Ab, depending on the condition of the host immune system (7).

Previous reports $(1,4-6,8,9)$ have demonstrated that positive Ap53Ab expression was detected in 24.0-33.1\% of CRC patients. These reports suggested that the measurement of Ap53Ab was useful for the screening of CRC patients with early-stage (stage 0 and I) disease, as the positive rate of Ap53Ab expression was higher compared with that of CEA and CA19-9. However, a consensus on the correlation between Ap53Ab expression and poor survival rate was not obtained $(4,8,10)$. The aim of the present study was to investigate the positive rate and clinical significance of Ap53Ab expression in $674 \mathrm{CRC}$ patients, and elucidate the association between Ap53Ab expression and survival.

\section{Patients and methods}

Patients and clinical procedures. The subjects included 674 CRC patients (237 women and 437 men), who were 
primarily diagnosed with CRC at the Saitama Medical Center (Kawagoe, Japan) between January 2010 and December 2014. A total of 115 healthy volunteers were also selected among patients who had been diagnosed with hemorrhoids or inguinal hernia at the Saitama Medical Center between October 2008 and July 2010. The median age of CRC patients and healthy volunteers was 69 years (range, 25-92 years) and 58 years (range, 16-84 years), respectively. Of the 674 patients investigated, the cancer localization was as follows: Cecum, $n=45$; ascending colon, $\mathrm{n}=99$; transverse colon, $\mathrm{n}=56$; descending colon, $n=24$; sigmoid colon, $n=173$; and rectum, $n=277$. The histological diagnosis was well-differentiated adenocarcinoma in 153 patients, moderately differentiated adenocarcinoma in 463 , poorly differentiated adenocarcinoma in 32 , mucinous adenocarcinoma in 17 , and other types in 8 patients. The carcinomas at the time of primary tumor resection were staged according to the Union for International Cancer Control classification (11) as follows: Stage 0, n=38; stage I, n=130; stage II, $\mathrm{n}=206$; stage III, $\mathrm{n}=194$; and stage IV, $\mathrm{n}=106$. Of the 194 patients with stage III CRC, $107(55.2 \%)$ received mFOLFOX6 or XELOX chemotherapy and 32 patients (16.5\%) received capecitabine or tegafur-uracil and leucovorin (UFT/LV) for 6 months as postoperative adjuvant chemotherapy. The mFOLFOX6 regimen comprises intravenous infusion of oxaliplatin $\left(85 \mathrm{mg} / \mathrm{m}^{2}\right)$ and $\mathrm{LV}\left(200 \mathrm{mg} / \mathrm{m}^{2}\right)$ for $2 \mathrm{~h}$, followed by rapid intravenous bolus infusion of 5-fluorouracil (5-FU; $400 \mathrm{mg} / \mathrm{m}^{2}$ ) for $5 \mathrm{~min}$, and continuous intravenous infusion of 5 -FU $\left(2,400 \mathrm{mg} / \mathrm{m}^{2}\right)$ for $46 \mathrm{~h}$. This regimen was repeated every 2 weeks. The XELOX regimen was administered as follows: Oxaliplatin $\left(130 \mathrm{mg} / \mathrm{m}^{2}\right)$ was injected intravenously. From day 1 to day 14 , capecitabine $\left(2,000 \mathrm{mg} / \mathrm{m}^{2} /\right.$ day $)$ was orally administered. Each cycle was repeated every 3 weeks. The capecitabine or UFT/LV group received 8 cycles of oral capecitabine $\left(2,400 \mathrm{mg} / \mathrm{m}^{2}\right.$ for 14 days followed by a 7-day rest per cycle), or 5 cycles of adjuvant UFT/LV (UFT $300 \mathrm{mg} / \mathrm{m}^{2}$ and LV $75 \mathrm{mg} /$ day for 28 days followed by a 7-day rest per cycle), respectively.

The present study was performed in accordance with the ethical guidelines for clinical research with the approval of our Institutional Ethics Committee. Informed consent was obtained from all individuals included in the study.

Measurement of Ap53Ab, CEA and CA19-9. Total blood samples were routinely collected from CRC patients and healthy volunteers at the time of diagnosis. The serum samples were processed using the MESACUP anti-p53Ab Test ELISA kit (MBL, Nagoya, Japan). The cut-off level of the serum Ap53Ab was set to $1.3 \mathrm{U} / \mathrm{ml}$ according to the manufacturer's instructions. The minimum value of Ap53Ab was reported at $<0.69 \mathrm{U} / \mathrm{ml}$; thus, values $<0.69$ were set to 0.69 for the analysis. The measurement of serum CEA and CA19-9 was also performed using ELISA. The normal level of CEA and CA19-9 was $<6.7 \mathrm{ng} / \mathrm{ml}$ and $<37.0 \mathrm{U} / \mathrm{ml}$, respectively.

Statistical analysis of Ap53Ab and CEA half-life. The half-life of Ap53Ab and CEA was calculated based on the following formula: $\mathrm{T}=\operatorname{loge} 2 \times \mathrm{xt} /(\log \mathrm{N} \mathrm{N}-\operatorname{loge} \mathrm{N})=0.693 \times \mathrm{xt} / 2.303(\log$ $\mathrm{N} 0-\log \mathrm{N}$ ), where T, half-life time (days); t, days from the operation to next blood testing; N0, preoperative value of
Ap53Ab or CEA; and N, postoperative value of Ap53Ab or CEA.

Of the Ap53Ab-positive patients with values $>10 \mathrm{U} / \mathrm{ml}$, 39 patients were selected. In addition, 19 patients with a CEA level elevated to $>20 \mathrm{ng} / \mathrm{ml}$ were selected. These CRC patients underwent curative surgery, with no evidence of recurrence during the 5-year follow-up. The measurement of Ap53Ab and CEA was performed within 60 days after surgery.

Statistical analysis. The Mann-Whitney U-test, Fisher's exact probability test and Chi-squared test were used where applicable. Survival analysis was conducted using the Kaplan-Meier method. The log-rank test was used to determine the significance of the survival curves. The period of disease-free survival (DFS) was calculated from the time of surgery to the time to recurrence and overall survival (OS) was calculated from the time of surgery to death from any cause. DFS and OS were censored at the time of the last visit to our hospital, or December 2015, whichever came first. Differences were considered statistically significant when $\mathrm{P}<0.05$. All statistical analyses were performed using a statistical software package (StatFlex ver.6.0; Artec, Osaka, Japan).

\section{Results}

Positive rates of tumor markers, including Ap53Ab, CEA and CA19-9. Of the 674 CRC patients, 195 (28.9\%) were positive for Ap53Ab, while 12 positive cases (10.4\%) were identified in the control group (Table I). The mean level \pm standard deviation (SD) of Ap53Ab in CRC patients and the control group was $26.0 \pm 132.0$ and $1.24 \pm 2.42 \mathrm{U} / \mathrm{ml}$, respectively (Table I). There difference in the Ap53Ab level between CRC patients and the control group was significant $(\mathrm{P}<0.0001$; Table I).

The positive rates of Ap53Ab, CEA, and CA19-9 in each CRC stage were as follows: Stage 0: 5.3, 10.5 and 7.9\%, respectively; stage I: $20.0,13.1$ and $12.3 \%$, respectively; stage II: 31.6 , 38.3 and $12.1 \%$, respectively; stage III: $34.0,43.8$ and $12.4 \%$, respectively; and stage IV: $34.0,79.2$ and $43.4 \%$, respectively (Table II). Positivity for Ap53Ab alone was observed in 94 patients (13.9\%; Table II). The positive rate of any examined markers was $58.7 \%$ (Table II).

Half-life time of Ap53Ab and CEA. The mean \pm SD half-life of Ap53Ab was 30.7 \pm 27.2 days (range, 8.5-118.1 days) in 39 CRC patients, while that of CEA was $11.3 \pm 4.4$ days (range, 4.4-21.8 days) in 19 CRC patients. Of the 39 patients with elevated Ap53Ab level, the level returned to normal within 1 year in only 4 patients $(10.3 \%)$.

Association between Ap53Ab expression and clinicopathological characteristics and prognosis. Positive expression of Ap53Ab was significantly associated with the depth of tumor invasion $(\mathrm{P}<0.001)$, lymph node metastasis $(\mathrm{P}=0.024)$, stage $(\mathrm{P}<0.001)$ and CEA level $(\mathrm{P}=0.005)$ (Table III). There was no association of Ap53Ab expression with gender, age, tumor location, histology, lymphatic invasion, venous invasion, liver metastasis or recurrence (Table III).

Regarding DFS in CRC patients with stage I-III disease, no significant difference was observed between patients with 
Table I. Ap53 Ab level and positive rate in colorectal cancer patients and healthy control group.

\begin{tabular}{lccc}
\hline Groups & $\mathrm{n}$ & Positive expression, n (\%) & Mean \pm SD (U/ml) \\
\hline Control & 115 & $12(10.4)$ & $1.24 \pm 2.42$ \\
Colorectal cancer & 674 & $195(28.9)$ & $26.0 \pm 132.0$ \\
\hline
\end{tabular}

Ap53Ab, anti-p53 antibody; SD, standard deviation.

Table II. Positive rate of tumor markers in 674 colorectal cancer patients.

\begin{tabular}{|c|c|c|c|c|c|c|}
\hline \multirow[b]{2}{*}{ Markers } & \multicolumn{6}{|c|}{ Stage, n (\%) } \\
\hline & $0(n=38)$ & $\mathrm{I}(\mathrm{n}=130)$ & II (n=206) & III $(n=194)$ & IV $(n=106)$ & Total $(n=674)$ \\
\hline Ap53Ab & $2(5.3)$ & $26(20.0)$ & $65(31.6)$ & $66(34.0)$ & $36(34.0)$ & 195 (28.9) \\
\hline CEA & $4(10.5)$ & $17(13.1)$ & $79(38.3)$ & $85(43.8)$ & $84(79.2)$ & 269 (39.9) \\
\hline CA19-9 & $3(7.9)$ & $16(12.3)$ & $25(12.1)$ & $24(12.4)$ & $46(43.4)$ & $114(16.9)$ \\
\hline Ap53Ab alone & $1(2.6)$ & $20(15.4)$ & $33(16.0)$ & $32(16.5)$ & $8(7.5)$ & 94 (13.9) \\
\hline Any marker & 7 (18.4) & $49(37.7)$ & $120(58.3)$ & $123(63.4)$ & $94(88.7)$ & $393(58.7)$ \\
\hline
\end{tabular}

Ap53Ab, anti-p53 antibody; CEA, carbohydrate antigen; CA19-9, carbohydrate antigen 19-9.

positive Ap53Ab expression $(n=157)$ and those with negative Ap53Ab expression $(n=373)(P=0.86$, Fig. 1). Moreover, no association between Ap53Ab expression and OS was observed ( $\mathrm{P}=0.44$, Fig. 2).

\section{Discussion}

In the present study, positive Ap53Ab expression was detected in $28.9 \%$ of 674 CRC patients. Previous studies $(1,4-6,8,9)$ reported a positive Ap53Ab expression rate of $24.0-33.1 \%$ in CRC patients. Our data were consistent with those reports in terms of the positive Ap53Ab rate. Previous reports $(4,5)$ suggested that the measurement of Ap53Ab was useful for the screening of CRC patients with early-stage (stage 0 and I) disease, since the positive rate of Ap53Ab expression was higher compared with that of CEA and CA19-9. There was an advantage of Ap53Ab for the detection of CRC patients with stage I disease, while the positive rate was low in CRC patients with stage 0 disease. When stage 0 and I patients were collectively analyzed, the positive rates of Ap53Ab, CEA and CA19-9 expression were $16.7,12.5$ and $11.3 \%$, respectively. The number of studies investigating Ap53Ab expression in a large population of stage 0 and I CRC patients is limited. A recent report (4) demonstrated that positive Ap53Ab expression was detected in $72(15.1 \%)$ of $478 \mathrm{CRC}$ patients with stage 0 and I disease. Another study (5) reported that 9 of 38 CRC patients $(23.7 \%)$ with stage I disease exhibited positive Ap53Ab expression. In our series, the positive rate of Ap53Ab expression in stage 0 and I and only stage I cases was 16.7 and $20.0 \%$, respectively, while that of CEA in stage 0 and I and only stage I cases was 12.5 and $13.1 \%$, respectively. Therefore, the positive rate of Ap53Ab expression was higher compared with that of CEA in CRC patients with early-stage disease.
Of note, one in three CRC patients with stage 0 and I disease may be detected using the combination of Ap53Ab, CEA and CA19-9. Of 168 patients with stage 0 and I disease, any one of these markers was positive in 56 patients $(33.3 \%)$. Since measurement of CEA alone was able to detect $12.5 \%$ of CRC cases, the detection rate with measurement of all markers is approximately three times higher compared with that of CEA alone.

The positive rate of Ap53Ab expression in stage II, III, and IV CRC is similar (31.6, 34.0 and $34.0 \%$, respectively). A similar result was also reported by Yamaguchi et al (4). Thus, it was hypothesized that repeated exposure to the p53 antigen may have reduced the production of Ap53Ab in the serum via induction of immunological tolerance (12). Positive expression of Ap53Ab alone was observed in 94 patients (13.9\%). This rate was similar with previously reported rates of 13.6 and $15 \%(4,5)$. Consequently, the combined measurement of Ap53Ab, CEA and CA19-9 improved the diagnosis of CRC up to $\sim 60 \%$.

Based on a previous study (1), the cut-off value was defined as $<1.3 \mathrm{U} / \mathrm{ml}$. The upper values of Ap53Ab were 4.39 and $16.9 \mathrm{U} / \mathrm{ml}$ in 205 healthy control donors and 189 patients with benign disease, respectively (1). In the present study, positive Ap53Ab expression was observed in 12 (10.4\%) of 115 healthy volunteers using the same detection method. The range of the Ap53Ab level in those volunteers was 1.5-19.5 U/ml. The 12 volunteers with positive Ap53Ab expression were followed up for $\sim 5$ years, and there was no cancer occurrence during that time. Previous reports indicated that false-positives may occur as a result of a severely dysplastic gastric mucosa (13), or lung tissues with squamous metaplasia and dysplasia (14). When the Ap53Ab value is as high as $20 \mathrm{U} / \mathrm{ml}$, a thorough physical examination should be 
Table III. Clinicopathological correlation of Ap53Ab expression in CRC.

\begin{tabular}{|c|c|c|c|}
\hline \multirow[b]{2}{*}{ Characteristics } & \multicolumn{2}{|c|}{$\begin{array}{c}\mathrm{Ap} 53 \mathrm{Ab} \\
\text { expression, } \mathrm{n}(\%)\end{array}$} & \multirow[b]{2}{*}{ P-valu } \\
\hline & $\begin{array}{l}\text { Positive } \\
(n=195)\end{array}$ & $\begin{array}{c}\text { Negative } \\
(n=479)\end{array}$ & \\
\hline Gender & & & 0.33 \\
\hline Male & $121(62.1)$ & $316(66.0)$ & \\
\hline Female & $74(37.9)$ & $163(34.0)$ & \\
\hline Age (years) & & & 0.42 \\
\hline$<70$ & $104(53.3)$ & $239(49.7)$ & \\
\hline$\geq 70$ & $91(46.7)$ & $240(50.3)$ & \\
\hline Tumor location & & & 0.53 \\
\hline Right & $55(28.2)$ & $145(30.3)$ & \\
\hline Left & $63(32.3)$ & $134(28.0)$ & \\
\hline Rectum & $77(39.5)$ & $200(41.8)$ & \\
\hline Differentiation & & & 0.16 \\
\hline High & $34(17.4)$ & $119(24.8)$ & \\
\hline Moderate & $144(73.9)$ & $319(66.6)$ & \\
\hline Poor & $11(5.6)$ & $21(4.4)$ & \\
\hline Mucinous & $6(3.1)$ & $11(2.3)$ & \\
\hline Others & $0(0.0)$ & $8(1.9)$ & \\
\hline Depth & & & $<0.00$ \\
\hline Tis & $2(1.0)$ & $36(7.5)$ & \\
\hline $\mathrm{T} 1$ & $13(6.7)$ & $64(13.4)$ & \\
\hline $\mathrm{T} 2$ & $24(12.3)$ & $59(12.3)$ & \\
\hline $\mathrm{T} 3$ & $103(52.8)$ & $207(43.2)$ & \\
\hline $\mathrm{T} 4$ & $8(4.1)$ & $103(21.5)$ & \\
\hline Unknown & $4(2.1)$ & $010(2.1)$ & \\
\hline Lymphatic invasion & & & 0.1 \\
\hline Absent & $79(40.5)$ & $227(47.4)$ & \\
\hline Present & $111(56.9)$ & $240(50.1)$ & \\
\hline Unknown & $5(2.6)$ & $12(2.5)$ & \\
\hline Venous invasion & & & 0.15 \\
\hline Absent & $59(30.2)$ & $173(36.1)$ & \\
\hline Present & $131(67.2)$ & $295(61.6)$ & \\
\hline Unknown & $5(2.6)$ & $11(2.3)$ & \\
\hline Lymph node metastasis & & & 0.02 \\
\hline Negative & $99(50.8)$ & $289(59.8)$ & \\
\hline Positive & $93(47.7)$ & $184(38.1)$ & \\
\hline Unknown & $3(1.5)$ & $10(2.1)$ & \\
\hline Liver metastasis & & & 0.28 \\
\hline Absent & $169(86.7)$ & $429(89.6)$ & \\
\hline Present & $26(13.3)$ & $50(10.4)$ & \\
\hline UICC stage & & & $<0.00$ \\
\hline 0 & $2(1.0)$ & $36(7.5)$ & \\
\hline $\mathrm{I}$ & $26(13.3)$ & $104(21.8)$ & \\
\hline II & $65(33.3)$ & $141(29.4)$ & \\
\hline III & $66(33.9)$ & $128(26.7)$ & \\
\hline IV & $36(18.5)$ & $70(14.6)$ & \\
\hline CEA & & & 0.005 \\
\hline$\leq 6.7$ & $101(51.8)$ & $303(63.3)$ & \\
\hline
\end{tabular}

Table III. Continued.

\begin{tabular}{lccc}
\hline & \multicolumn{3}{c}{ Ap53Ab } \\
& \multicolumn{2}{c}{ expression, $\mathrm{n}(\%)$} & \\
\cline { 2 - 3 } Characteristics & $\begin{array}{c}\text { Positive } \\
(\mathrm{n}=195)\end{array}$ & $\begin{array}{c}\text { Negative } \\
(\mathrm{n}=479)\end{array}$ & P-value \\
\hline$>6.7$ & $94(48.2)$ & $175(36.5)$ & \\
Unknown & $0(0.0)$ & $1(0.2)$ & 0.45 \\
Recurrence & & & \\
Absent & $139(87.4)$ & $368(90.0)$ & \\
Present & $18(11.3)$ & $38(9.3)$ & \\
Unknown & $2(1.3)$ & $3(0.7)$ & \\
\hline
\end{tabular}

Bold print indicates statistical significance. CRC, colorectal cancer; Ap53Ab, anti-p53 antibody; UICC, Union for International Cancer Control; CEA, carbohydrate antigen.

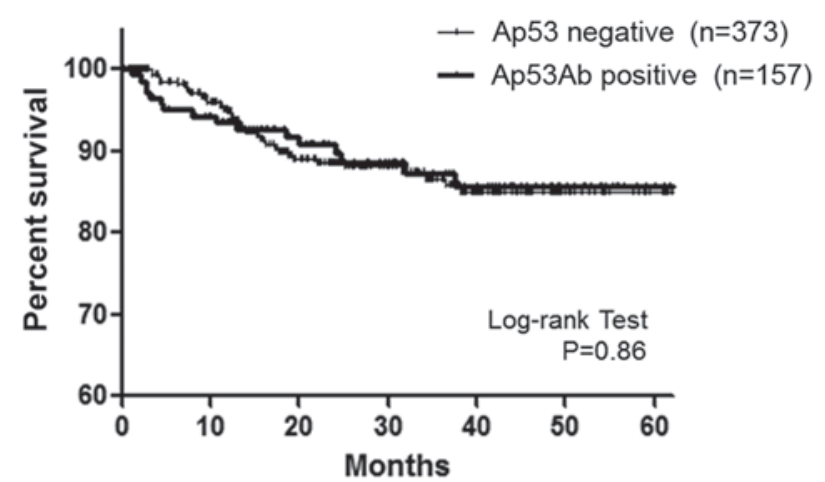

Figure 1. Comparison of disease-free survival between colorectal cancer patients with positive $(n=157)$ and those with negative $(n=373)$ anti-p53 antibody (Ap53Ab) expression.

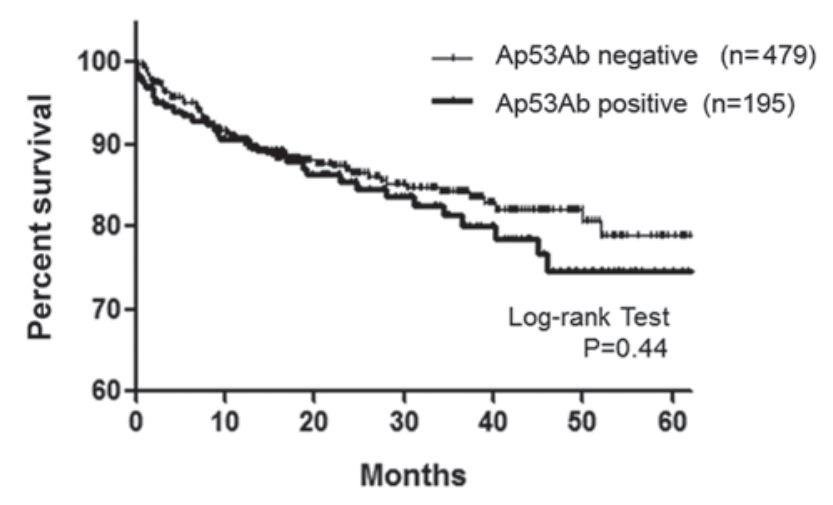

Figure 2. Comparison of overall survival between colorectal cancer patients with positive $(n=195)$ and those with negative $(n=479)$ anti-p53 antibody (Ap53Ab) expression.

performed, bearing in mind the possibility of false-positive readings. Of note, when positive Ap53Ab expression is detected in clinical practice, physical examination should be performed to rule out the possibility of various cancers, including head and neck, esophageal, uterine, breast, 
prostatic, biliary tract, lung, bladder, gastric and pancreatic cancer (1).

In the present study, the Ap53Ab level decreased slowly following curative resection. Ap53Ab is an IgG antibody and the half-life of $\mathrm{IgG}$ is considered to be 21 days (15). The mean half-life of CEA was reported to be 4-12 days, depending on the status of recurrence $(16,17)$. In our results, the mean $\pm \mathrm{SD}$ half-life of Ap53Ab was 30.7 \pm 27.2 days in 39 CRC patients, while that of CEA was $11.3 \pm 4.4$ days in 19 CRC patients. A previous study (18) reported that the Ap53Ab level decreased to normal following curative resection within at least 6 months in almost all Ap53Ab-positive CRC patients. Recently, Kawahara et al (19) reported data supporting our results, as the positive Ap53Ab rate was $75 \%$ at 6 months, $70.8 \%$ at 12 months, and $54.2 \%$ at 24 months after curative operation in 24 CRC patients with no recurrence. In our results, the Ap53Ab level had returned to normal within 12 months in only 4 (10.3\%) of the 39 CRC patients with elevated Ap53Ab level, whereas in some cases without recurrence it took $\sim 5$ years to return to the normal range. Therefore, the measurement of Ap53Ab should be limited to prior to surgery. When the elevated Ap53Ab level decreases slowly, even if it requires a long time, it does not necessarily indicate that the Ap53Ab level reflects the presence of residual tumor and/or recurrence.

It has been reported that positive expression of Ap53Ab was significantly associated with lymph node metastasis and lymphatic invasion (4,5,20). Furthermore, Yamaguchi et al (4) reported that other factors, including tumor location, histology, depth of tumor invasion, vessel invasion, distant metastasis, CEA and recurrence, were also significantly associated with positive Ap53Ab expression. Other studies $(9,10)$ reported that no significant correlation was observed between positive

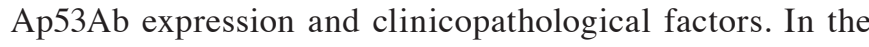
present study, several factors, including depth of tumor invasion, lymph node metastasis, stage and CEA, were found to be significantly associated with positive Ap53Ab expression. The association between elevated Ap53Ab and lymph node metastasis was consistent with previous data from an Asian population (4,5,7). In gastric cancer, an elevated Ap53Ab level tended to be associated with lymph node metastasis (21), but positive Ap53Ab expression was not found to be correlated with lymph node metastasis in esophageal squamous cell carcinoma (22). Therefore, the elevated Ap53Ab level may be consistently associated with deeper depth of invasion and lymph node metastasis in CRC, that is to say that deeper depth of invasion and lymph node metastasis may be involved in the production of Ap53Ab.

In the present study, no correlation was observed between Ap53Ab expression and poor survival rate, including DFS and OS. Although previous reports $(8,23)$ demonstrated that positive Ap53Ab expression was associated with poor prognosis in CRC patients, there appears to be no consensus $(4,6,10)$. Recently, Yamaguchi et al (4) reported that no association between Ap53Ab expression and OS was found in 1384 CRC patients, although Ap53Ab expression was associated with relapse-free survival in 1212 CRC patients who underwent curative surgery. Of those 1212 CRC patients, 339 (28\%) received adjuvant chemotherapy. In our series, $>70 \%$ of CRC patients with stage III disease were treated with adjuvant chemotherapy. Of those patients, $77 \%$ underwent oxaliplatin-based adjuvant chemotherapy, including mFOLFOX6 and XELOX. It may be hypothesized that oxaliplatin-based chemotherapy may improve the prognosis of CRC patients regardless of Ap53Ab expression. Several researchers have analyzed the prognosis of cancer patients with TP53 expression using immunohistochemical examination and genetic testing (6,24-26). Since the association between TP53 expression and prognosis using has not yet reached a firm conclusion, even with these methods, it is difficult to predict prognosis using Ap53Ab expression. Chang et al (6) investigated genetic alteration of TP53, overexpression of intratumoral p53 protein and Ap53Ab expression in $\mathrm{CRC}$, and found that the positive rates of each examination were $56.3,44.9$ and $28.1 \%$, respectively; they concluded that only genetic alterations of TP53 were significantly associated with poor prognosis, while intratumoral TP53 and Ap53Ab expression were not. Moreover, they mentioned that TP53 mutations at exons 6 and 7 were associated with the presence of Ap53Ab. The frequency of CRC patients with positive Ap53Ab expression was estimated at $\sim 50 \%$ among CRC patients with genetic alterations of TP53. Further studies are required to validate the association between Ap53Ab expression and survival.

Taken together, our results indicate that the measurement of Ap53Ab may contribute to the detection of early-stage CRC in clinical practice. The use of Ap53Ab with CEA and CA19-9 may increase the diagnostic yield for CRC up to $\sim 60 \%$. Furthermore, the time to normalization of the Ap53Ab level was longer than expected in CRC patients with elevated Ap53Ab level preoperatively. Ap53Ab expression was associated with lymph node metastasis, although the association between Ap53Ab expression and poor prognosis could not be fully elucidated.

\section{References}

1. Shimada H, Ochiai T and Nomura F; Japan 533 Antibody Research Group: Titration of serum p53 antibodies in 1,085 patients with various types of malignant tumors: A multiinstitutional analysis by the Japan p53 Antibody Research Group. Cancer 97: 682-689, 2003.

2. Shimada H, Takeda A, Arima M, Okazumi S, Matsubara H, Nabeya Y, Funami Y, Hayashi H, Gunji Y, Suzuki T, et al: Serum p53 antibody is a useful tumor marker in superficial esophageal squamous cell carcinoma. Cancer 89: 1677-1683, 2000.

3. Yamamoto S, Chishima T, Adachi S, Harada F, Toda Y, Arioka H, Hasegawa N and Kakuta Y: Serum p53 antibody in breast cancer. Cancer Biomark 14: 203-206, 2014.

4. Yamaguchi T, Takii Y and Maruyama S: Usefulness of serum p53 antibody measurement in colorectal cancer: An examination of 1384 primary colorectal cancer patients. Surg Today 44: 1529-1535, 2014.

5. Ochiai H, Ohishi T, Osumi K, Tokuyama J, Urakami H, Seki S, Shimada A, Matsui A, Isobe Y, Murata Y, et al: Reevaluation of serum p53 antibody as a tumor marker in colorectal cancer patients. Surg Today 42: 164-168, 2012.

6. Chang SC, Lin JK, Lin TC and Liang WY: Genetic alteration of p53, but not overexpression of intratumoral p53 protein, or serum p53 antibody is a prognostic factor in sporadic colorectal adenocarcinoma. Int J Oncol 26: 65-75, 2005.

7. Tang R, Ko MC, Wang JY, Changchien CR, Chen HH, Chen JS, Hsu KC, Chiang JM and Hsieh LL: Humoral response to p53 in human colorectal tumors: A prospective study of 1,209 patients. Int J Cancer 94: 859-863, 2001

8. Shiota G, Ishida M, Noguchi N, Oyama K, Takano Y, Okubo M, Katayama S, Tomie Y, Harada K, Hori K, et al: Circulating p53 antibody in patients with colorectal cancer: Relation to clinicopathologic features and survival. Dig Dis Sci 45: 122-128, 2000. 
9. Noaki R, Kawahara H, Watanabe K, Ushigome T, Kobayashi S and Yanaga K: Serum p53 antibody is a useful tumor marker of early colorectal cancer. Int Surg 95: 287-292, 2010.

10. Suppiah A, Alabi A, Madden L, Hartley JE, Monson JR and Greenman J: Anti-p53 autoantibody in colorectal cancer: Prognostic significance in long-term follow-up. Int J Colorectal Dis 23: 595-600, 2008.

11. Sobin LH, Gospodarowicz MK and Wittekind C (eds): TNM classification of malignant tumours, 7th edition, 2010.

12. Takeda A, Shimada H, Nakajima K, Yoshimura S, Suzuki T, Asano T, Ochiai T and Isono K: Serum p53 antibody as a useful marker for monitoring of treatment of superficial colorecta adenocarcinoma after endoscopic resection. Int J Clin Oncol 6 : 45-49, 2001.

13. Joypaul BV, Newman EL, Hopwood D, Grant A, Qureshi S Lane DP and Cuschieri A: Expression of p53 protein in normal, dysplastic and malignant gastric mucosa: An immunohistochemical study. J Pathol 170: 279-283, 1993.

14. Bennett WP, Colby TV, Travis WD, Borkowski A, Jones RT, Lane DP, Metcalf RA, Samet JM, Takeshima Y, Gu JR, et al: p53 protein accumulates frequently in early bronchial neoplasia. Cancer Res 53: 4817-4822, 1993.

15. Morell A, Terry WD and Waldmann TA: Metabolic properties of IgG subclasses in man. J Clin Invest 49: 673-680, 1970.

16. Ito K, Hibi K, Ando H, Hidemura K, Yamazaki T, Akiyama S and Nakao A: Usefulness of analytical CEA doubling time and half-life time for overlooked synchronous metastases in colorectal carcinoma. Jpn J Clin Oncol 32: 54-58, 2002.

17. Choi JS and Min JS: Significance of postoperative serum level of carcinoembryonic antigen (CEA) and actual half life of CEA in colorectal cancer patients. Yonsei Med J 38: 1-7, 1997.

18. Takeda A, Shimada H, Nakajima K, Imaseki H, Suzuki T, Asano T, Ochiai T and Isono K: Monitoring of p53 autoantibodies after resection of colorectal cancer: Relationship to operative curability. Eur J Surg 167: 50-53, 2001.
19. Kawahara H, Watanabe K, Enomoto H, Toyama Y, Akiba T and Yanaga K: Normalization of serum p53 antibody levels in patients after curative resection for colorectal cancer. Anticancer Res 33: 2221-2225, 2013.

20. Nozoe T, Yasuda M, Honda M, Inutsuka $S$ and Korenaga D: Clinicopathologic significance in serum presence of anti-p53 antibody in patients with colorectal carcinoma. Hepatogastroenterology 54: 1422-1425, 2007.

21. Nakajima K, Suzuki T, Shimada H, Hayashi H, Takeda A and Ochiai T: Detection of preoperative serum anti-p53 antibodies in gastric cancer. Tumour Biol 20: 147-152, 1999.

22. Shimada H, Nakajima K, Ochiai T, Koide Y, Okazumi SI, Matsubara H, Takeda A, Miyazawa Y, Arima M and Isono K: Detection of serum p53 antibodies in patients with esophageal squamous cell carcinoma: Correlation with clinicopathologic features and tumor markers. Oncol Rep 5: 871-874, 1998.

23. Kressner U, Glimelius B, Bergström R, Påhlman L, Larsson A and Lindmark G: Increased serum p53 antibody levels indicate poor prognosis in patients with colorectal cancer. $\mathrm{Br} \mathrm{J}$ Cancer 77 : 1848-1851, 1998

24. Morikawa T, Kuchiba A, Liao X, Imamura Y, Yamauchi M, Qian ZR, Nishihara R, Sato K, Meyerhardt JA, Fuchs CS and Ogino S: Tumor TP53 expression status, body mass index and prognosis in colorectal cancer. Int J Cancer 131: 1169-1178, 2012.

25. Russo A, Bazan V, Iacopetta B, Kerr D, Soussi T and Gebbia N; TP53-CRC Collaborative Study Group: The TP53 colorectal cancer international collaborative study on the prognostic and predictive significance of p53 mutation: Influence of tumor site, type of mutation and adjuvant treatment. J Clin Oncol 23: 7518-7528, 2005.

26. Munro AJ, Lain S and Lane DP: P53 abnormalities and outcomes in colorectal cancer: A systematic review. Br J Cancer 92: 434-444, 2005. 